\title{
Differential effects of Th17 cytokines during the response of neutrophils to Burkholderia cenocepacia outer membrane protein A
}

\author{
SOAD NADY', MONA ABDEL-RAHMAN', SILVIA A. SOUSA², JORGE H. LEITĂO', \\ MOSTAFA MORAD ${ }^{l}$, REHAB E. EL-HENNAMY ${ }^{1}$ \\ 'Department of Zoology and Entomology, Faculty of Science, Helwan University, Helwan, Egypt \\ ${ }^{2}$ Department of Bioengineering, IBB - Institute for Bioengineering and Biosciences, Instituto Superior Técnico, University of Lisbon, \\ Lisbon, Portugal
}

\begin{abstract}
Thelper 17 cells are involved in the immunopathology of cystic fibrosis. They play a key role in recruitment of neutrophils, which is the first line of defence against bacteria. Additionally, Burkholderia cenocepacia outer membrane protein A (OmpA) BCAL2958 is considered a potential protective epitope for vaccine development. The present study aimed to investigate the neutrophil response to OmpA in the presence of Th17 cytokines, IL-17 and IL-22 at different times of activation. Neutrophils were isolated from whole blood of healthy volunteers and activated with OmpA in the presence of IL-17, IL-22 or both cytokines together. Supernatant was collected after $1 \mathrm{~h}, 2 \mathrm{~h}, 4 \mathrm{~h}, 8 \mathrm{~h}$, and $12 \mathrm{~h}$. Neutrophil activation was assessed by measuring MPO, TNF- $\alpha$, elastase, hydrogen peroxide, catalase and NO. The results revealed that the combination of $I L-17$ and $I L-22$ cytokines induced the release of $N E$, catalase, $\mathrm{H}_{2} \mathrm{O}_{2}$ and TNF- $\alpha$ from neutrophils activated with Burkholderia OmpA at late stages of activation. However, IL-22 alone or IL-17 alone decreased the myeloperoxidase (MPO), catalase and NE levels at early stages of neutrophil activation. The presence of IL-17 alone led to a significant increase in $T N F-\alpha$ level after $1 \mathrm{~h}$ and $12 \mathrm{~h}$. However, the presence of IL-22 alone led to a significant increase in TNF- $\alpha$ level after only $1 \mathrm{~h}$ but a significant decrease after $8 \mathrm{~h}$ of activation was observed as compared to OmpA stimulated neutrophils. In conclusion, Th17 cytokines $I L-17$ and IL-22, have differential effects during the neutrophil response to Burkholderia OmpA.
\end{abstract}

Key words: Burkholderia cepacia complex (BCC), outer membrane protein A (OmpA), neutrophils, Thelper 17 cytokines.

(Centr Eur Immunol 2019; 44 (4): 403-413)

\section{Introduction}

Outer membrane proteins (OMPs) are predominant antigens in Gram-negative bacteria [1]. OmpA proteins, a sub-class of OMPs, contribute to the ability of Gram-negative bacteria to invade mammalian cells [2]. On the other hand, OMPs are major surface antigens of Burkholderia cepacia complex (Bcc) and are considered potential protective epitopes for vaccine development [3]. The presence of antibodies against the OmpA-like protein BCAL2958 in serum samples from cystic fibrosis $(\mathrm{CF})$ patients with a clinical record of respiratory infection by $B$. cepacia complex bacteria was recently reported [4]. Furthermore, the purified BCAL2958 protein was demonstrated to stimulate neutrophils in vitro [4].

Neutrophils are the first line of immune defence and have a primary role in resistance against extracellular pathogens and in acute inflammation [5]. Neutrophils can kill pathogens by both oxidative (respiratory burst) and non-oxidative (lytic or proteolytic enzymes) mechanisms $[5,6]$. Oxygen-independent mechanisms involve releasing myeloperoxidase (MPO) and neutrophil elastase, which are the most common antimicrobial effectors in disruption of the bacterial cell membrane integrity and degradation of proteolytic bacterial virulence factors [7]. The oxygen-dependent mechanisms involve generation of reactive oxygen species (ROS) and upon activation of neutrophils, NADPH-oxidase complexes assemble and transfer electrons to molecular oxygen producing superoxide $\left(\mathrm{O}_{2}^{-}\right)$. Superoxide dismutates spontaneously to hydrogen peroxide, which in turn is the substrate for MPO to form hypochlorous acid (HOCL), which is the most bactericidal antioxidant in neutrophils [8]. Furthermore, as reported by previous studies, TNF- $\alpha$, which is also produced by

Correspondence: Soad Nady, PhD, Department of Zoology and Entomology, Helwan University, Ain Helwan, 11790 Helwan, Egypt, e-mail: soadnady@science.helwan.edu.eg Submitted: 17.05.2017; Accepted: 28.08.2017 
neutrophils, plays one of the major biological roles in the host defence against bacterial, viral and parasitic infections $[9,10]$.

Recently, T helper 17 (Th17) cells were reported to play a key role in the host defence against extracellular microbes such as bacteria and fungi, as well as other inflammatory responses [11]. Th17 cells secrete mainly interleukin-17 (IL-17) and IL-22 [12]. Recent studies reported that Th17 cells can orchestrate the neutrophil response against extracellular bacteria [13]. Additionally, IL-17A is reported to recruit neutrophils during the chronic inflammatory response in lung disorders but its effect on neutrophil activity is controversial [14].

In $\mathrm{CF}$, pulmonary disease is the major cause of morbidity and mortality and is caused by a vicious cycle of infection and inflammation and dysregulation of cytokines [15]. Th17 cytokines have been linked to the pulmonary exacerbations and neutrophilia in CF disease [16]. Little is known about the direct effect of Th17 cytokines on neutrophil activity. The aim of the present study was to investigate the functional activity of neutrophils during their response to B. cenocepacia OmpA in the presence of Th17 cytokines IL-17 and IL-22 after different times of activation.

\section{Material and methods}

\section{Purification of OmpA-like protein}

The Burkholderia cenocepacia J2315 BCAL2958 outer membrane protein A (OmpA) used in this study was produced and purified by affinity chromatography as previously described by Sousa et al. (2016).

\section{Subjects}

The blood samples of 10 adult healthy male volunteers (mean age $30 \pm 5$ years) were collected. Participants were among a group of controls of an ongoing study (STDF, 1814) approved by the research ethics committee of Cairo University. All participants gave informed consent before participation in the study.

\section{Isolation and purification of neutrophils from whole blood}

Neutrophils were isolated from EDTA anti-coagulated venous blood as described previously [6] with some modifications. Briefly, the blood was centrifuged using a Ficoll Hypaque (Amersham Pharmacia, Piscataway, NJ) density gradient at $400 \times \mathrm{g}$ for $25 \mathrm{~min}$ to remove the mononuclear cells. Then, red blood cells (RBCs) were lysed using ACK lysis buffer (Sigma, St. Louis, MO). Resulting neutrophil preparations were $>98 \%$ pure as assessed by flow cytometry. More than $95 \%$ of the neutrophils were viable as measured by trypan blue. Purified neutrophils were washed and re-suspended in DMEM medium (Sigma, St. Louis, $\mathrm{MO})$ at the appropriate concentration.

\section{Activation of neutrophils with OmpA in the presence of $\mathrm{Th} 17$ cytokines}

Isolated neutrophils $\left(1 \times 10^{5}\right.$ cells/well) were activated with OmpA ( $2 \mu \mathrm{g} / \mathrm{ml}$, as optimized $)$ in the presence or absence of Th17 cytokines, IL-17 (125 pg/ml, as optimized) and IL-22 (300 pg/ml, as optimized). $100 \mathrm{ng} / \mathrm{ml} \mathrm{[17]} \mathrm{li-}$ popolysaccharide (Sigma-Aldrich, Germany) was used as a positive control. Supernatant was collected after $1 \mathrm{~h}, 2 \mathrm{~h}$, $4 \mathrm{~h}, 8 \mathrm{~h}$, and $12 \mathrm{~h}$ for measuring MPO, TNF- $\alpha$, elastase, hydrogen peroxide and catalase using ELISA as well as NO by Griess reagent. Non-stimulated neutrophils were used as negative controls.

\section{Measurements of neutrophil mediators}

\section{Nitric oxide}

Nitric oxide was assessed using Griess reagent according to [18].

\section{Hydrogen peroxide $\left(\mathrm{H}_{2} \mathrm{O}_{2}\right)$}

Hydrogen peroxide $\left(\mathrm{H}_{2} \mathrm{O}_{2}\right)$ was measured using a colorimetric kit (Bio-diagnostic, Egypt) according to [19].

\section{Catalase}

Catalase was measured using a colorimetric kit (Biodiagnostic, Egypt) according to [19].

\section{ELISA measured mediators}

Human myeloperoxidase, tumour necrosis factor- $\alpha$ and neutrophil elastase were measured using an MPO ELISA kit (Booster Immuno-reader, USA), human TNF- $\alpha$ ELISA kit (Booster Immuno-reader, USA) and elastase ELISA kit (ASSAY PRO, USA), respectively, according to the manufacturer's instructions.

\section{Statistical analysis}

Statistical analysis was performed using Graph Pad Prism software. ANOVA was used to analyse the data among different treatments at each time point. The data obtained were represented as mean \pm SD. Results with a $p$ value $<0.05$ were considered significant.

\section{Results}

In our previous study, the effect of Burkholderia cenocepacia OmpA on normal neutrophils as compared to non-activated neutrophils was reported [4]. In the current study, the effect of Th17 cytokines on neutrophils stimulated with OmpA was investigated and compared to neutrophils activated with OmpA alone. 
Effect of Burkholderia cenocepacia OmpA on tumour necrosis factor-alpha (TNF- $\alpha$ ) released from neutrophils in the presence of Th17 cytokines

Stimulation of neutrophils with OmpA in the presence of IL-17 induced a significant ( $p=0.0269$ and $p=0.0299$ ) increase in TNF- $\alpha$ level after $1 \mathrm{~h}$ and $12 \mathrm{~h}$ respectively as compared to those activated with OmpA alone (Fig. 1A). However, in the presence of IL-22 a significant $(p=0.0384)$ increase in TNF- $\alpha$ level after $1 \mathrm{~h}$ but significant $(p=0.0355)$ decrease after $8 \mathrm{~h}$ of activation were observed as compared to OmpA stimulated neutrophils (Fig. 1B). A significant ( $p=0.0198$ and $p=0.0453$ ) increase in TNF- $\alpha$ level after $1 \mathrm{~h}$ and $12 \mathrm{~h}$ respectively were observed in the presence of both IL-17 with IL-22 as compared to OmpA activated neutrophils (Fig. 1C).

\section{Effect of Burkholderia cenocepacia OmpA on elastase released from neutrophils in the presence of Th17 cytokines}

Neutrophils stimulated with OmpA in the presence of both cytokines together showed a significant increase in

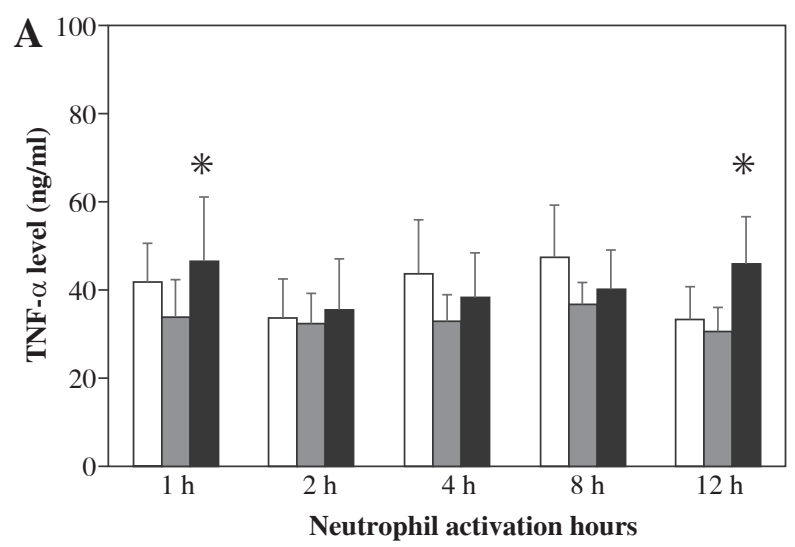

$\square$ Omp A $\square$ IL-17 $\square$ Omp A + IL-17

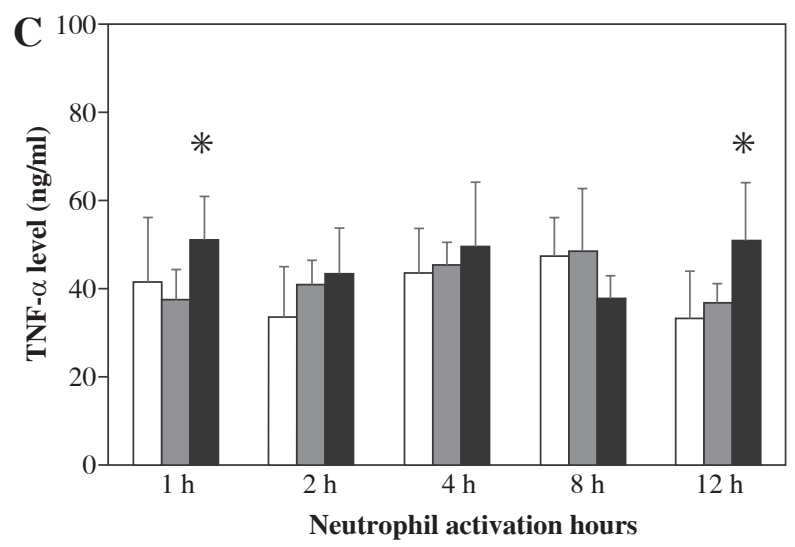

$\square$ Omp A $\square \mathrm{IL}-17$ + IL-22 $\square$ Omp A + IL-17+ IL-22 the concentration of elastase after $1 \mathrm{~h}$ and $12 \mathrm{~h}(p=0.0178$ and $p=0.0249$ respectively) as compared to OmpA alone stimulated neutrophils (Fig. 2C).

In contrast, as compared to OmpA activated neutrophils a significant decrease $(p=0.0280)$ in elastase level was observed in the presence of IL-22 after $4 \mathrm{~h}$ (Fig. 2B). However, no significant change in elastase level was observed in the presence of IL-17 (Fig. 2A).

\section{Effect of Burkholderia cenocepacia OmpA on catalase released from neutrophils in the presence of $\mathrm{Th} 17$ cytokines}

As shown in Fig. 3A, in the presence of IL-17, there was a significant decrease $(p<0.05)$ in the concentration of catalase produced by neutrophils activated with OmpA after $1 \mathrm{~h}, 2 \mathrm{~h}, 4 \mathrm{~h}$ and $12 \mathrm{~h}$ but an increase after $8 \mathrm{~h}$ $(p<0.05)$. Also, presence of IL-22 induced a significant decrease after $1 \mathrm{~h}, 2 \mathrm{~h}$ and $4 \mathrm{~h}$ as compared to OmpA activated neutrophils (Fig. 3B). Similar to the effect of IL-17 alone, presence of both cytokines, IL-17 with IL-22, induced a significant decrease in catalase level after $1 \mathrm{~h}$,

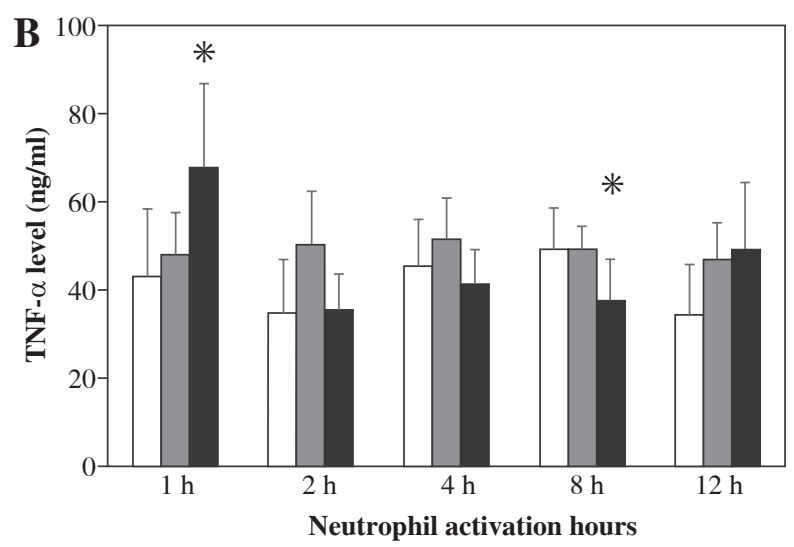

$\square$ Omp A $\square$ IL-22 $\square$ Omp A + IL-22

Fig. 1. Effect of Burkholderia cenocepacia outer membrane protein A on tumour necrosis factor-alpha released from neutrophils in the presence of Th17 cytokines; IL-17 (A), IL-22 (B) and both IL-17 plus IL-22 (C). Bars represent mean $\pm \mathrm{SD}$ of 8 experiments. * significant different as compared to OmpA at $p<0.05$ 

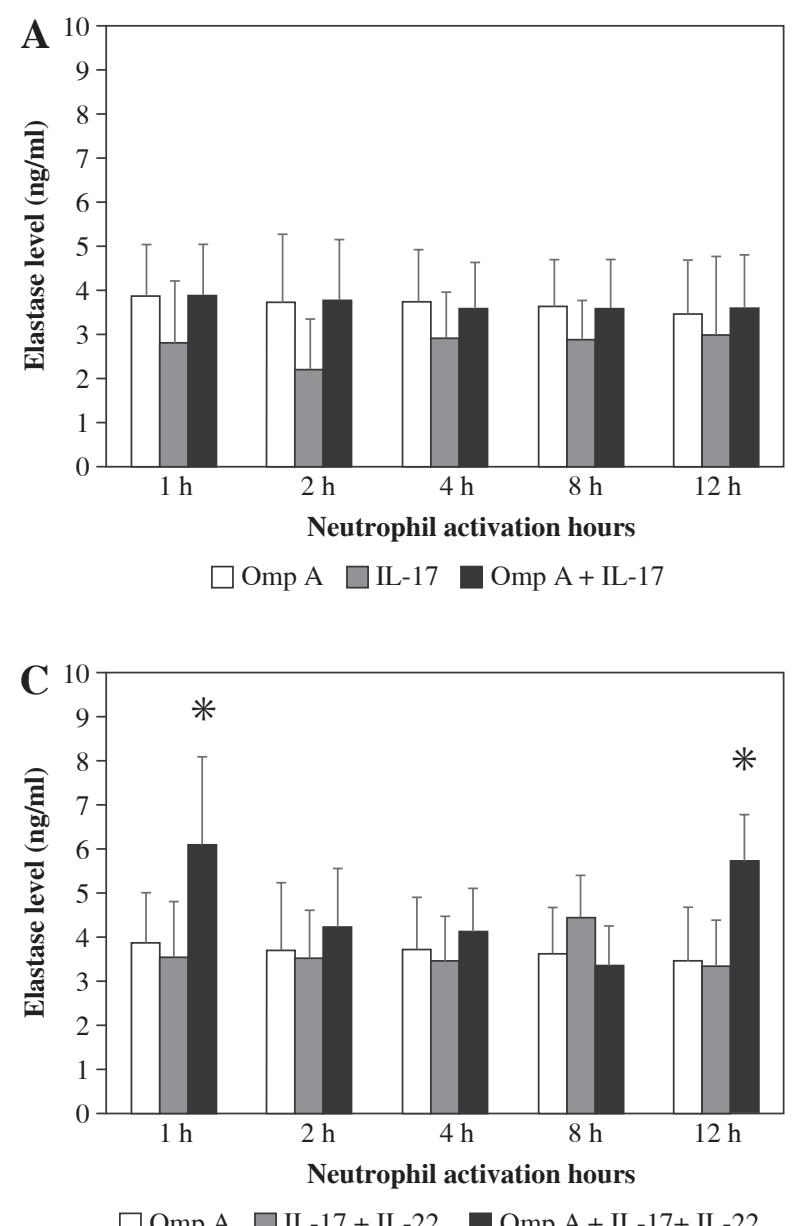

$2 \mathrm{~h}, 4 \mathrm{~h}$ and $12 \mathrm{~h}$ but a significant increase $(p<0.05)$ after $8 \mathrm{~h}$ as compared to OmpA activated neutrophils (Fig. 3C).

\section{Effect of Burkholderia cenocepacia OmpA on myeloperoxidase (MPO) released from neutrophils in the presence of Th17 cytokines}

The release of MPO from neutrophils stimulated with OmpA decreased after $2 \mathrm{~h}$ significantly ( $p=0.0225$ and $p=0.0189$ respectively) in the presence of IL-17 alone (Fig. 4A) or IL-22 alone (Fig. 4B) as compared to OmpA activated neutrophils. However, no significant change in MPO level was observed in the presence of IL-17 with IL-22 (Fig. 4C).

\section{Effect of Burkholderia cenocepacia OmpA on hydrogen peroxide $\left(\mathrm{H}_{2} \mathrm{O}_{2}\right)$ released from neutrophils in the presence of Th17 cytokines}

There were no significant changes in $\mathrm{H}_{2} \mathrm{O}_{2}$ production by neutrophils activated with OmpA in the presence of IL-17 alone or IL-22 alone (Figs. 5A and B). Howev$\mathrm{er}$, in the presence of both cytokines together a significant

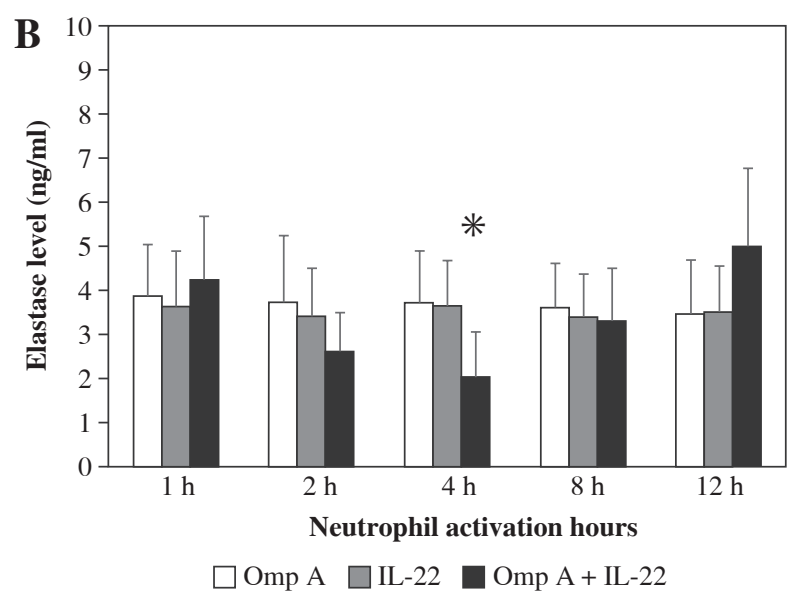

Fig. 2. Effect of Burkholderia cenocepacia outer membrane protein A on elastase released from neutrophils in the presence of Th17 cytokines; IL-17 (A), IL-22 (B) and both IL-17 plus IL-22 (C). Bars represent mean \pm SD of 8 experiments. * significant different as compared to OmpA at $p<0.05$

$(p=0.0465)$ level of hydrogen peroxide was observed after $8 \mathrm{~h}$ of neutrophil activation with OmpA as compared to neutrophils activated with OmpA without cytokines (Fig. 5C).

\section{Effect of Burkholderia cenocepacia OmpA on nitric oxide (NO) released from neutrophils in the presence of Th17 cytokines}

There were no significant changes in NO production by neutrophils activated with OmpA in the presence of IL-17, IL-22 or both cytokines together as compared to OmpA activated neutrophils (Fig. 6).

\section{Discussion}

Respiratory infections caused by bacteria of the Burkholderia cepacia complex (Bcc) are life-threatening to cystic fibrosis patients and no vaccines are available to prevent these devastating infections [20]. Colonization by Bcc usually occurs on the respiratory mucosa, so development of a mucosal vaccine may be of a great value, as this route of vaccination is associated with both mucosal and systemic immunity $[3,21]$. OMPs are major surface antigens 


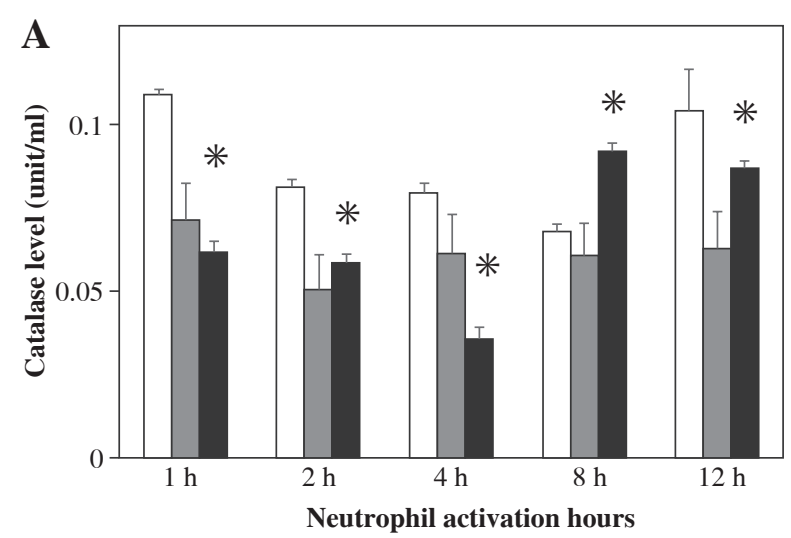

$\square$ Omp A $\square$ IL-17 $\square$ Omp A + IL-17

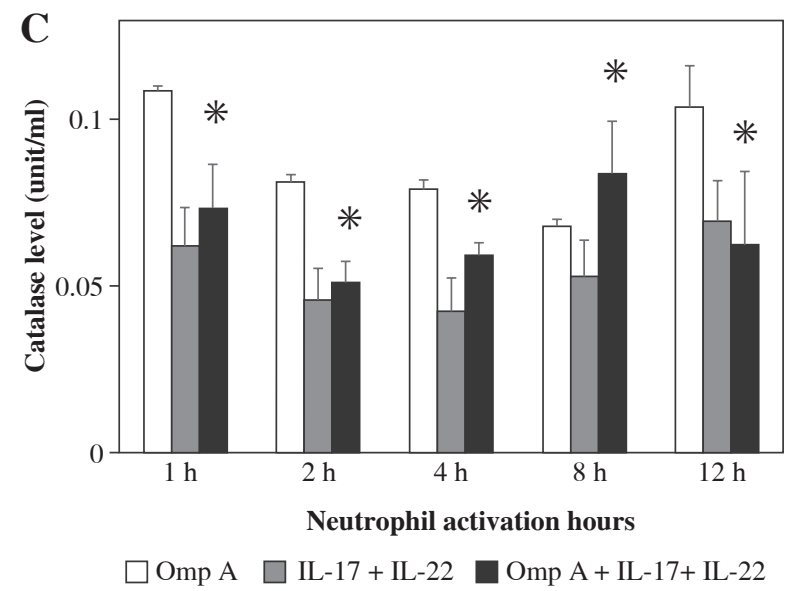

of Bcc and are considered potential protective epitopes for vaccine development [3].

Neutrophils are essential components in the body's innate immune defence and play a crucial role in phagocytosis and subsequent destruction of invading microorganisms [22]. In cystic fibrosis, inflammation is caused by persistent bacterial infections in the lung and is characterized by the persistent infiltration of massive numbers of neutrophils, which leads to lung injury [14]. Recently, Th17 cells were reported to play a role in neutrophil function during the response to extracellular bacteria [23].

To accomplish bacterial killing, neutrophils utilize their powerful antibacterial mediators consisting of toxic ROS and antibacterial proteins [24]. Neutrophil-derived ROS are produced by the action of NADPH oxidase, a membrane-bound enzyme complex that ferries electrons from cytoplasmic NADPH across the membrane to molecular oxygen that is converted into superoxide anion $\left(\mathrm{O}_{2}^{-}\right)$, which rapidly dismutates to oxygen and hydrogen peroxide $\left(\mathrm{H}_{2} \mathrm{O}_{2}\right.$ ) according to the reaction $2 \mathrm{O}_{2}^{-}+2 \mathrm{H}^{+} \rightarrow \mathrm{O}_{2}+\mathrm{H}_{2} \mathrm{O}_{2}$ [25]. Once $\mathrm{H}_{2} \mathrm{O}_{2}$ is formed, it can be involved in many reactions that enhance its toxicity [26].

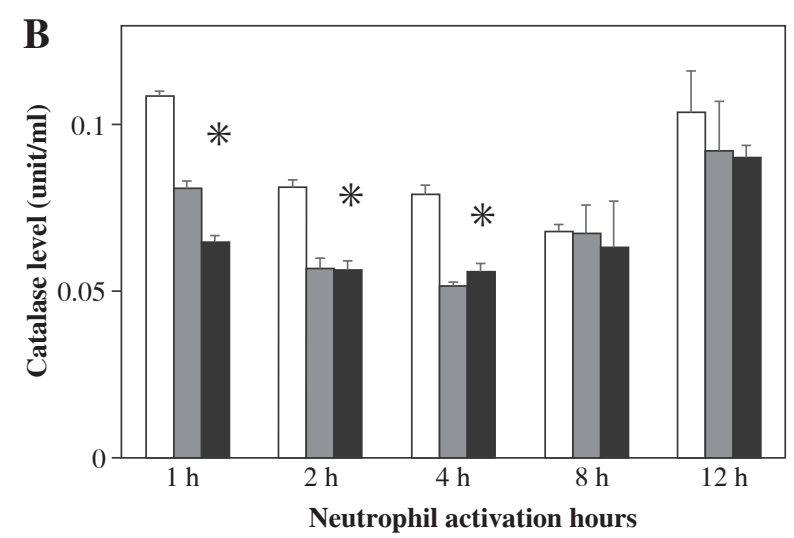

$\square$ Omp A $\square$ IL-22 $\square$ Omp A + IL-22

Fig. 3. Effect of Burkholderia cenocepacia outer membrane protein A on catalase released from neutrophils in the presence of Th17 cytokines; IL-17 (A), IL-22 (B) and both IL-17 plus IL-22 (C). Bars represent mean \pm SD of 8 experiments. * significant different as compared to OmpA at $p<0.05$

In the present study, neither IL-17 nor IL-22 cytokines had a significant effect on peroxide production by neutrophils after all activation times. These results are in agreement with those of Pelletier et al. [23] who found that IL-17 has no effect on peroxide production by neutrophils activated with formyl-methionyl-leucyl phenylalanine (fMLP) for up to $2 \mathrm{~h}$ of activation. Regarding IL-22, some previous studies [21, 27] indicate its involvement in the response against bacterial infections by inducing the release of other innate immune mediators, which may augment our finding.

Catalase is one of the key antioxidant enzymes involved in the protection against immune infection and oxidative stress [28]. Phagocytic neutrophils usually produce catalase under heavy oxidative stress resulting from high levels of hydrogen peroxide. Catalase is produced to protect phagocytic neutrophils and tissues against oxidative damage by $\mathrm{H}_{2} \mathrm{O}_{2}$. Catalase represents the first mechanism which can decompose $\mathrm{H}_{2} \mathrm{O}_{2}$ into water and oxygen according to the reaction; $2 \mathrm{H}_{2} \mathrm{O}_{2} \rightarrow 2 \mathrm{H}_{2} \mathrm{O}+\mathrm{O}_{2}$ [29].

The results of the current study revealed that catalase release was decreased in the presence of Th17 cytokines 

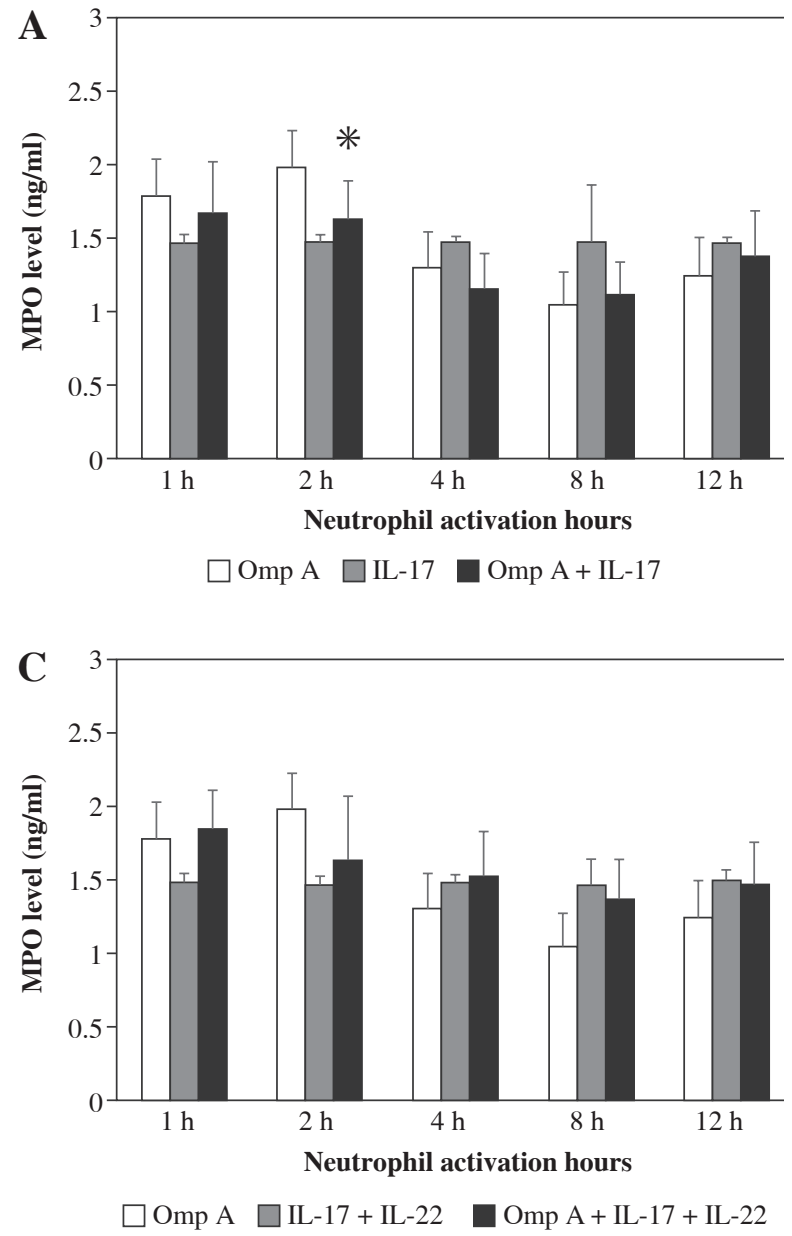

(IL-17, IL-22 or both cytokines) after $1 \mathrm{~h}, 2 \mathrm{~h}, 4 \mathrm{~h}$ and $12 \mathrm{~h}$ as compared to those observed in the absence of cytokines. However, an increase in the concentration of catalase was observed in the presence of IL-17 and both IL-17 and IL-22 together after $8 \mathrm{~h}$ as compared to OmpA stimulated neutrophils. This increase was parallel to the increase of $\mathrm{H}_{2} \mathrm{O}_{2}$ level observed almost around the same time point of neutrophil activation especially in the presence of both IL-17 and IL-22. These results may indicate that Th17 cytokines at certain time points of activation have an inflammatory role in the neutrophil response and they have a protective role at other time points depending on other mediators released to the microenvironment during the response.

These observations are supported by the study of Klebanoff et al. [30], who found that enzymes such as catalase, which consumes $\mathrm{H}_{2} \mathrm{O}_{2}$, can protect certain organisms from the toxic effect of neutrophils in vitro. Additionally, Jansen et al. [26] indicated that upon infection of Caenorhabditis elegans, S. pyogenes produces hydrogen peroxide that can kill the host, but catalase production by the host protects the host from such killing. Moreover, the oxida-

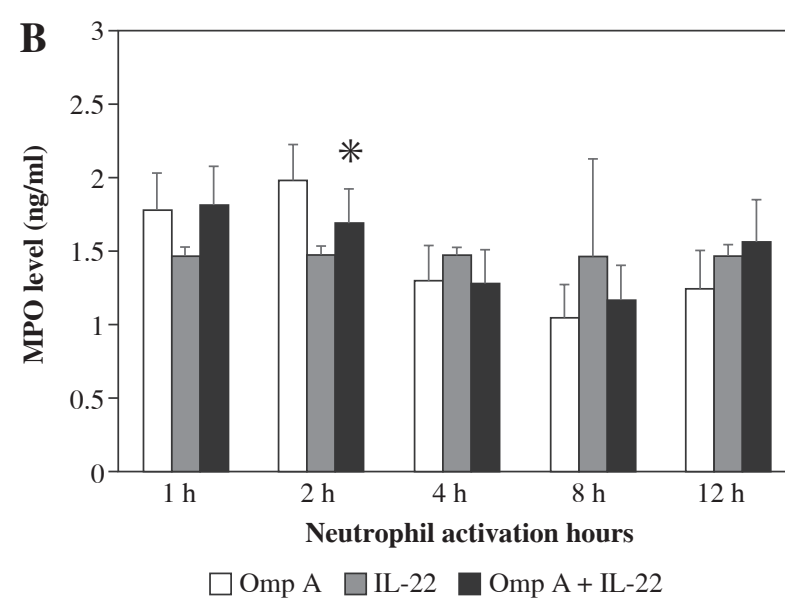

Fig. 4. Effect of Burkholderia cenocepacia outer membrane protein A on myeloperoxidase released from neutrophils in the presence of Th17 cytokines; IL-17 (A), IL-22 (B) and both IL-17 plus IL-22 (C). Bars represent mean \pm SD of 8 experiments. * significant different as compared to OmpA at $p<0.05$

tive pathway involves release of nitric oxide (NO), which is generated either by the constitutively expressed enzymes nitric oxide synthase (NOS)-1 and NOS-3 or the induced enzyme NOS-2. NOS-2 is not expressed in naive cells but is induced by immunological stimuli such as bacterial lipopolysaccharide (LPS) or cytokines such as tumour necrosis factor (TNF)- $\alpha$ [31].

A few studies have discussed the role of different cytokines in nitric oxide production from neutrophils. For instance, stimulation of neutrophils with a mixture of interleukin 1 (IL-1), TNF- $\alpha$ and interferon gamma (IFN- $\gamma$ ) was reported to possibly enhance iNOS mRNA and protein, one of the enzymes responsible for NO production [32].

In the present study, neither the individual cytokine IL-17 nor IL-22 induced NO production by neutrophils activated with OmpA throughout the different activation times, which suggests that these cytokines did not enhance the inducible NO synthase mRNA production. These results may also provide an explanation for the raised inflammatory and damage effects for the presence of Th17 cell cytokines in many diseases such as cystic fibrosis and lung disease [33]. Lack of NO production was associated with 


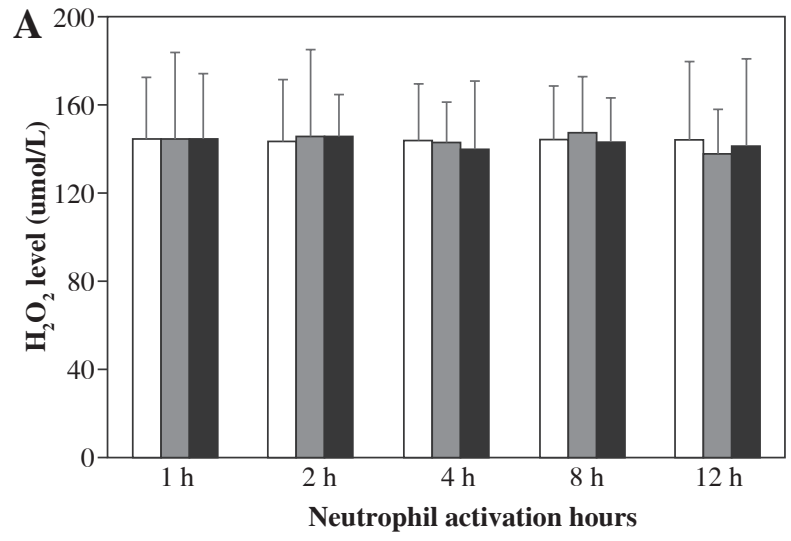

$\square$ Omp A $\square$ IL-17 $\square$ Omp A + IL-17

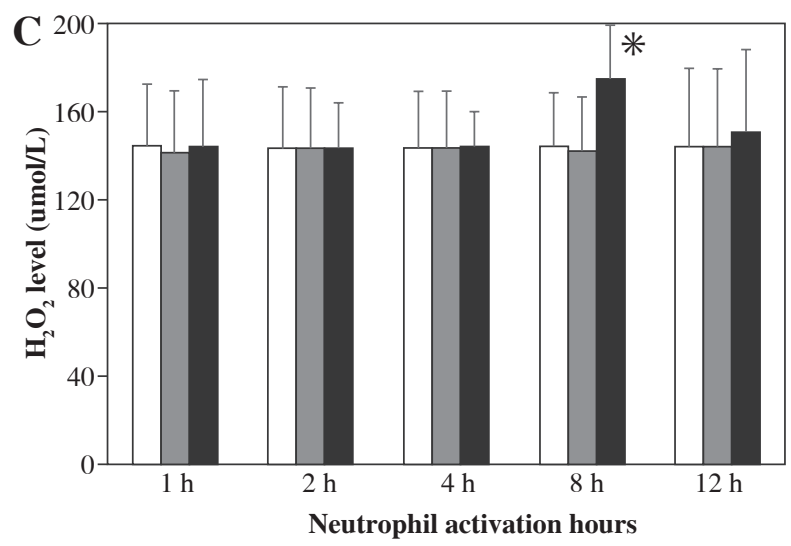

$\square$ Omp A $\square \mathrm{IL}-17$ + IL-22 $\square$ Omp A + IL-17+ IL-22

extensive damage, including increased bacterial growth, increased apoptosis, and the exacerbation of histopathological characteristics in mouse livers infected with Salmonella enterica serovar Typhimurium [34]. NO can be used in killing of pathogenic bacteria, including Burkholderia mallei, B. cepacia, and Pseudomonas aeruginosa [35].

In contrast to the current results, a recent study demonstrated that cytokine-treated human neutrophils contain iNOS mRNA and protein that produces nitrotyrosine around ingested bacteria, most likely from the production of peroxynitrite, a highly reactive anion that may contribute to microbial and tissue killing [30]. Additionally, peroxynitrite may be involved in induction of apoptosis in isolated human neutrophils [36].

It is well known that neutrophil azurophilic granules contain a rich supply of the green haem enzyme myeloperoxidase, which in combination with $\mathrm{H}_{2} \mathrm{O}_{2}$ and chloride constitutes a potent anti-microbial system [37]. The results of the present study showed a significant decrease of MPO activity in the presence of IL-17 alone or IL-22 alone after $2 \mathrm{~h}$ as compared to OmpA stimulated neutrophils.

Several studies suggest that MPO can attack bacteria in different ways. MPO oxidizes or halogenates various

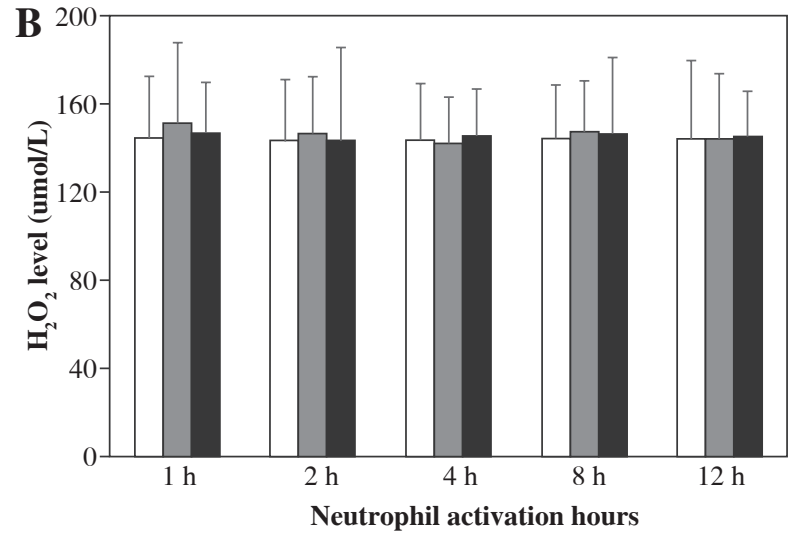

$\square$ Omp A $\square$ IL-22 $\square$ Omp A + IL-22

Fig. 5. Effect of Burkholderia cenocepacia outer membrane protein A on hydrogen peroxide released from neutrophils in the presence of Th17 cytokines; IL-17 (A), IL-22 (B) and both IL-17 plus IL-22 (C). Bars represent mean \pm SD of 8 experiments. * significant different as compared to OmpA at $p<0.05$

bacterial molecules. The bacterial wall might be the major target, which affects pathways associated with bacterial growth, such as energy production and DNA synthesis [38]. MPO might also contribute to the efficient killing by neutrophils by acting in conjunction with other antimicrobial molecules such as neutrophil elastase, which degrades the outer membrane proteins of bacteria [22] and may allow MPO to diffuse intracellularly and generate reactive intermediates to target molecules essential for bacterial survival. So, MPO oxidation of bacterial membrane proteins may enhance their degradation by NE [39]. Therefore, the inhibitory effect of IL-17 and IL-22 on MPO production by neutrophils activated with Burkholderia OmpA in the current study may be another cause of the pathological effects in CF patients infected with Bcc.

The other pathway of neutrophils to kill the invading microbes is the non-oxidative pathway that involves antibacterial proteins such as NE. In vitro studies showed that $\mathrm{NE}^{-/-}$neutrophils exhibited less killing activity than $\mathrm{NE}^{+/+}$ neutrophils [40]. A central role in the pathophysiology of CF has been attributed to NE; it causes a significant damage to the CF airway by degrading nearly all the structural proteins of the lung, including elastin, collagen type 

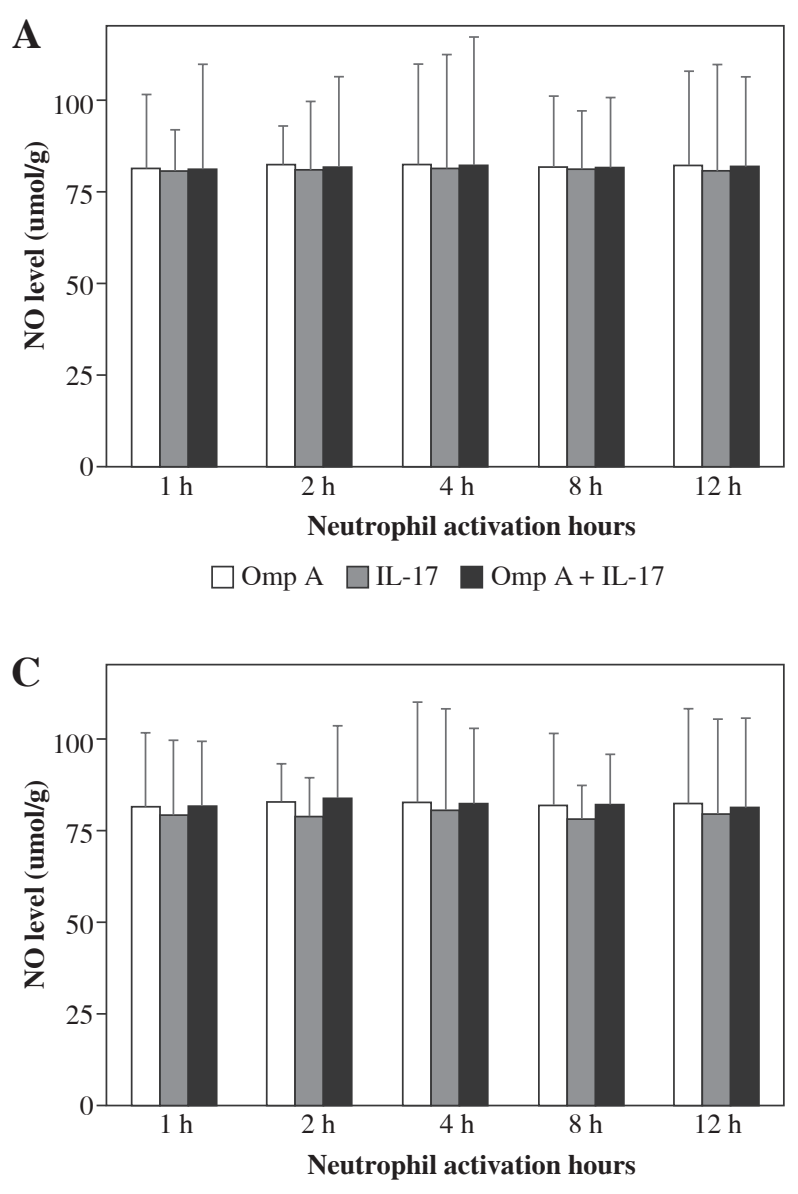

I-IV, fibronectin and proteoglycans [15]. Previous studies showed the importance of NE involvement in bacterial killing, but it also causes a defective role in the structural proteins of the lung [40-42].

Many studies have reported the antibacterial role of MPO and neutrophil elastase but few studies have investigated the effect of Th17 cytokines on MPO and NE release by neutrophils [13, 43, 44]. IL-17 is reported to play a central role in pulmonary host defence by recruiting and inducing the activity of neutrophils in the bronchoalveolar space [14]. IL-17 was also shown to potentially increase in association with neutrophilic inflammation and mucus excess, the two marked characteristics of CF lung disease, as well as dysregulation of acquired immunity [13, 43, 44].

The results of the present study revealed that IL-22 caused a decrease in MPO and NE release after $2 \mathrm{~h}$ and $4 \mathrm{~h}$ of neutrophil activation as compared to OmpA stimulated neutrophils. In contrast, these effects were not observed for IL-17. These results are partially similar to a previous study reporting that stimulation of rat neutrophils with human IL-17 did not increase MPO or elastase in vitro activity, while MPO and elastase activity in vivo increased, as well as the neutrophil count in bronchoalveolar lavage

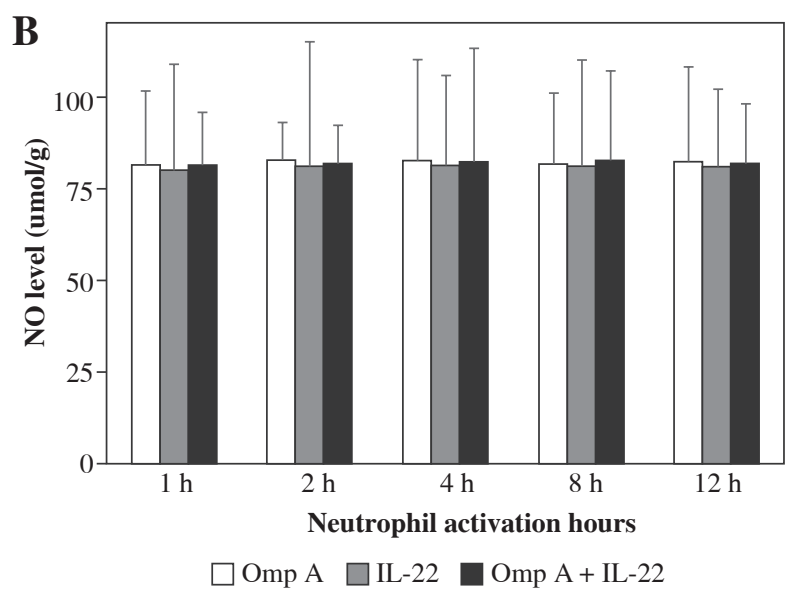

Fig. 6. Effect of Burkholderia cenocepacia outer membrane protein A (Omp A) on nitric oxide released from neutrophils in the presence of Th17 cytokines; IL-17 (A), IL-22 (B) and both IL-17 plus IL-22 (C). Bars represent mean \pm SD of 8 experiments

(BAL) fluid $6 \mathrm{~h}$ after intratracheal instillation [45]. These observations indicate that IL-17 cytokine can activate neutrophils, not only cause their recruitment into the airways in vivo. This effect is probably achieved through induced release of mediators by other airway cells in the microenvironment, suggesting that there is no direct effect exerted by IL-17 cytokine on neutrophils activation.

Neutrophil elastase has the ability to cause prolongation of the inflammatory process by degrading complement, releasing C5a, a potent chemo-attractant for neutrophils [42]. It was also found that repeated antigen stimulation in the lung leads to collagen deposition and the development of fibrosis. In the absence of IL-22 expression, mice had significantly more lung fibrosis than control mice, and the administration of recombinant cytokine further reduced the production of collagen. These data suggest that IL-22 cytokine plays a protective role against inflammation and development of fibrosis [46]. IL-22 is reported as a protective agent during hepatitis and inflammatory bowel disease [47] as well as against lung fibrosis [48] and liver fibrosis developed from Schistosoma infection [49].

Increased elastase release after $1 \mathrm{~h}$ and $12 \mathrm{~h}$ was observed using the combination of both IL-17 with IL-22 cy- 
tokines, in contrast to their effect on MPO release. These data also suggest that Th17 cells may be important for the host defence against extracellular pathogens.

TNF- $\alpha$ is important for the normal response to infection, although inappropriate or excessive production can be harmful. All known responses to TNF- $\alpha$ are triggered by binding to one of two distinct receptors designated TNFR1 and TNFR2, which are differentially regulated on various cell types in normal and diseased tissues $[9,10]$.

Preliminary evidence that Th17 cytokines may be involved in the pathogenesis of $\mathrm{CF}$ results from the fact that levels of IL-17 cytokine in sputum are raised in adult patients with $\mathrm{CF}$ during respiratory exacerbations and decline after antibiotic therapy [16]. In the current study, IL-17 cytokine alone or in combination with IL-22 potentiates the effect of OmpA on neutrophils to release marked levels of TNF- $\alpha$ after $1 \mathrm{~h}$ and $12 \mathrm{~h}$ of activation. These results are in agreement with other studies reporting that the main proinflammatory effects of IL-17 cytokine were mediated by induction of TNF- $\alpha$, IL-1 $\beta$, chemokines (CXCL8, CXCL1, CXCL10), GM-CSF, G-CSF, IL-6 [50, 51] and metalloproteases [52]. These findings may give an insight into the functional feature of IL-17 cytokine in the recruitment, activation, and migration of neutrophils [53]. Moreover, recent studies showed that IL-17 cytokine upregulates a number of gene products involved in cell activation, growth, and proliferation, including the proinflammatory cytokines IL-1 $\beta$ and TNF- $\alpha$ in activated macrophages $[54,55]$.

The observed increases in TNF- $\alpha$ levels in the present study may explain the role of IL-17 cytokine and TNF- $\alpha$ in many inflammatory lung diseases, including cystic fibrosis and chronic obstructive pulmonary disease, because TNF- $\alpha$ primes the neutrophil respiratory burst; upregulates the expression of adhesion molecules, cytokines and chemokines; and at high local concentrations can stimulate ROS production in adherent neutrophils $[9,56]$.

IL-22 cytokine was reported to have a role during inflammatory conditions. IL-22 cytokine can induce various tissue-specific genes, including serum amyloid A, anti-microbial proteins (b-defensin, Reg3 $\gamma$, lipocalin-2), and mucins $[27,57]$. In the lung, if mucin production was elevated, it would lead to many complications in cystic fibrosis patients as this increase gives an optimal environment for the growth of different bacterial species [58].

In the present study, IL-22 cytokine enhanced the release of TNF- $\alpha$ from neutrophils stimulated with OmpA after $1 \mathrm{~h}$ of activation, but there was a marked decrease after $8 \mathrm{~h}$. These results resemble the observations obtained by a previous study reporting that IL-22R activation drives signal transducer and activator of transcription (STAT-3) and the elevated transcription of IL- 8 and TNF- $\alpha$, concluding that IL-22 has both pro- and anti-inflammatory effects [59]. The significant increases of TNF- $\alpha$ levels in the current study may be explained on the basis of the dual nature of IL-22 cytokine, protective versus inflammatory, which depends on the inflammatory context that includes the duration and amount of IL-22 present in the microenvironment, the overall cytokine milieu as well as other tissues involved in the response [46].

A synergistic effect for both IL-17 with IL-22 cytokines on the production of TNF- $\alpha$ after $12 \mathrm{~h}$ of neutrophil activation with OmpA was observed in the current study. So, the present results may confirm that Th17 cells are implicated in the inflammatory response against Burkholderia OmpA by contributing to neutrophil activation.

Additionally, combination of IL-17 and IL-22 cytokines induced the release of NE, catalase, $\mathrm{H}_{2} \mathrm{O}_{2}$ and TNF- $\alpha$ from neutrophils activated with Burkholderia OmpA at late stages of activation. However, IL-22 alone or IL-17 alone decreased the MPO, catalase and NE levels at early stages of neutrophil activation. In conclusion, Th17 cytokines show differential effects on neutrophils activated with Burkholderia cenocepacia outer membrane protein A.

\section{Acknowledgments}

The authors would like to thank the Egyptian Science and Technology Development Fund (STDF) for supporting this study through grant No. 1814 awarded to Dr Soad Nady under the framework of Egypt/US cooperation program. Also, funding from the Portuguese Foundation for Science and Technology [grants UID/BIO/04565/2013 and PTDC/BIA-MIC/1615/2014 and the Post-doc grant SFRH/BPD/102006/2014 to SAS] and from Programa Operacional Regional de Lisboa 2020 [Project N. 007317] is acknowledged.

\section{The authors declare no conflict of interest.}

\section{References}

1. Koebnik R, Locher KP, Van Gelder P (2000): Structure and function of bacterial outer membrane proteins: barrels in a nutshell. Mol Microbiol 37: 239-253.

2. Weiser JN, Gotschlich EC (1991): Outer membrane protein A (OmpA) contributes to serum resistance and pathogenicity of Escherichia coli K-1. Infect Immun 59: 2252-2258.

3. Makidon PE, Knowlton J, Groom JV 2nd, et al. (2010): Induction of immune response to the $17 \mathrm{kDa}$ OMPA Burkholderia cenocepacia polypeptide and protection against pulmonary infection in mice after nasal vaccination with an OMP nanoemulsion-based vaccine. Med Microbiol Immunol 199: 81-92.

4. Sousa SA, Morad M, Feliciano JR, et al. (2016): The Burkholderia cenocepacia OmpA-like protein BCAL2958: identification, characterization, and detection of anti-BCAL2958 antibodies in serum from B. cepacia complex-infected cystic fibrosis patients. AMB Express 6: 41.

5. Nathan C (2006): Neutrophils and immunity: challenges and opportunities. Nat Rev Immunol 6: 173-182.

6. Costa D, Marques AP, Reis RL, et al. (2006): Inhibition of human neutrophil oxidative burst by pyrazolone derivatives. Free Radic Biol Med 40: 632-640. 
7. Scott DA, Krauss J (2012): Neutrophils in periodontal inflammation. Front Oral Biol 15: 56-83.

8. Urban CF, Reichard U, Brinkmann V, Zychlinsky A (2006): Neutrophil extracellular traps capture and kill Candida albicans yeast and hyphal forms. Cell Microbiol 8: 668-676.

9. Bradley JR (2008): TNF-mediated inflammatory disease. J Pathol 214: 149-160.

10. Petit-Bertron AF, Tabary O, Corvol H, et al. (2008): Circulating and airway neutrophils in cystic fibrosis display different TLR expression and responsiveness to interleukin-10. Cytokine 41: 54-60.

11. Rutitzky LI, Stadecker MJ (2006): CD4 T cells producing pro-inflammatory interleukin-17 mediate high pathology in schistosomiasis. Mem Inst Oswaldo Cruz 101 Suppl 1: 327 330.

12. Stockinger B, Veldhoen M (2007): Differentiation and function of Th17 T cells. Curr Opin Immunol 19: 281-286.

13. Cua DJ, Tato CM (2007): Innate IL-17-producing cells: the sentinels of the immune system. Nat Rev Immunol 10: 479489.

14. Linden A, Laan M, Anderson GP (2005): Neutrophils, interleukin-17A and lung disease. Eur Respir J 25: 159-172.

15. Hartl D, Latzin P, Hordijk P, et al. (2007): Cleavage of CXCR1 on neutrophils disables bacterial killing in cystic fibrosis lung disease. Nat Med 13: 1423-1430.

16. McAllister F, Henry A, Kreindler JL, et al. (2005): Role of IL$17 \mathrm{~A}, \mathrm{IL}-17 \mathrm{~F}$, and the IL-17 receptor in regulating growth-related oncogene-alpha and granulocyte colony-stimulating factor in bronchial epithelium: implications for airway inflammation in cystic fibrosis. J Immunol 175: 404-412.

17. El-Shorbagy A, Shata MT, Mohey MA, Nady S (2016): Differential effects of $t$ helper-17 cytokines on the functions of granulocytes isolated from schistosoma mansoni-infected patients and healthy individuals. J Arab Soc Med Res 11: 29-36.

18. Green LC, Wagner DA, Glogowski J, et al. (1982): Analysis of nitrate, nitrite, and [15N]nitrate in biological fluids. Anal Biochem 126: 131-138.

19. Fossati P, Prencipe L, Berti G (1980): Use of 3,5-dichloro-2-hydroxybenzenesulfonic acid/4-aminophenazone chromogenic system in direct enzymic assay of uric acid in serum and urine. Clin Chem 26: 227-231.

20. Choh LC, Ong GH, Vellasamy KM, et al. (2013): Burkholderia vaccines: are we moving forward? Front Cell Infect Microbiol 3: 5.

21. Aujla SJ, Chan YR, Zheng M, et al. (2008): IL-22 mediates mucosal host defense against Gram-negative bacterial pneumonia. Nat Med 14: 275-281.

22. Segal AW (2005): How neutrophils kill microbes. Annu Rev Immunol 23: 197-223.

23. Pelletier M, Maggi L, Micheletti A, et al. (2010): Evidence for a cross-talk between human neutrophils and Th17 cells. Blood 115: 335-343.

24. Mantovani A, Cassatella MA, Costantini C, Jaillon S (2011): Neutrophils in the activation and regulation of innate and adaptive immunity. Nat Rev Immunol 11: 519-531.

25. Fu H, Belaaouaj AA, Dahlgren C, Bylund J (2003): Outer membrane protein A deficient Escherichia coli activates neutrophils to produce superoxide and shows increased susceptibility to antibacterial peptides. Microbes Infect 5: 781-788.

26. Jansen WT, Bolm M, Balling R, et al. (2002): Hydrogen peroxide-mediated killing of Caenorhabditis elegans by Streptococcus pyogenes. Infect Immun 70: 5202-5207.
27. Zheng Y, Valdez PA, Danilenko DM, et al. (2008): Interleukin-22 mediates early host defense against attaching and effacing bacterial pathogens. Nat Med 14: 282-289.

28. Zhang Y, Fu D, Yu F, et al. (2011): Two catalase homologs are involved in host protection against bacterial infection and oxidative stress in Crassostrea hongkongensis. Fish Shellfish Immunol 31: 894-903.

29. Roos D, Weening RS, Wyss SR, Aebi HE (1980): Protection of human neutrophils by endogenous catalase: studies with cells from catalase-deficient individuals. J Clin Invest 65 : 1515-1522.

30. Klebanoff SJ, Kettle AJ, Rosen H, et al. (2013): Myeloperoxidase: a front-line defender against phagocytosed microorganisms. J Leukoc Biol 93: 185-198.

31. Coleman JW (2001): Nitric oxide in immunity and inflammation. Int Immunopharmacol 1: 1397-1406.

32. Evans TJ, Buttery LD, Carpenter A, et al. (1996): Cytokine-treated human neutrophils contain inducible nitric oxide synthase that produces nitration of ingested bacteria. Proc Natl Acad Sci U S A 93: 9553-9558.

33. Di Stefano A, Caramori G, Gnemmi I, et al. (2009): T helper type 17-related cytokine expression is increased in the bronchial mucosa of stable chronic obstructive pulmonary disease patients. Clin Exp Immunol 157: 316-324.

34. Alam MS, Akaike T, Okamoto S, et al. (2002): Role of nitric oxide in host defense in murine salmonellosis as a function of its antibacterial and antiapoptotic activities. Infect Immun 70: 3130-3142.

35. Jones-Carson J, Laughlin JR, Stewart AL, et al. (2012): Nitric oxide-dependent killing of aerobic, anaerobic and persistent Burkholderia pseudomallei. Nitric Oxide 27: 25-31.

36. Ward C, Wong TH, Murray J, et al. (2000): Induction of human neutrophil apoptosis by nitric oxide donors: evidence for a caspase-dependent, cyclic-GMP-independent, mechanism. Biochem Pharmacol 59: 305-314.

37. Klebanoff SJ (2005): Myeloperoxidase: friend and foe. J Leukoc Biol 77: 598-625.

38. Daugherty A, Dunn JL, Rateri DL, et al. (1994): Myeloperoxidase, a catalyst for lipoprotein oxidation, is expressed in human atherosclerotic lesions. J Clin Invest 94: 437-444.

39. Olszowska E, Olszowski S, Zgliczyński JM, et al. (1989): Enhancement of proteinase-mediated degradation of proteins modified by chlorination. Int J Biochem 21: 799-805.

40. Belaaouaj A (2002): Neutrophil elastase-mediated killing of bacteria: lessons from targeted mutagenesis. Microbes Infect 4: $1259-1264$.

41. Boggs DR (1967): The kinetics of neutrophilic leukocytes in health and in disease. Semin Hematol 4: 359-386.

42. Belaaouaj A, Kim KS, Shapiro SD (2000): Degradation of outer membrane protein A in Escherichia coli killing by neutrophil elastase. Science 289: 1185-1188.

43. Aujla SJ, Dubin PJ, Kolls JK (2007): Interleukin-17 in pulmonary host defense. Exp Lung Res 33: 507-518.

44. Bettelli E, Oukka M, Kuchroo VK (2007): T(H)-17 cells in the circle of immunity and autoimmunity. Nat Immunol 8: 345-350.

45. Hoshino H, Laan M, Sjöstrand M, et al. (2000): Increased elastase and myeloperoxidase activity associated with neutrophil recruitment by IL-17 in airways in vivo. J Allergy Clin Immunol 105 (1 Pt 1): 143-149.

46. Zenewicz LA, Flavell RA (2011): Recent advances in IL-22 biology. Int Immunol 23: 159-163. 
47. Zenewicz LA, Yancopoulos GD, Valenzuela DM, et al. (2007): Interleukin-22 but not interleukin-17 provides protection to hepatocytes during acute liver inflammation. Immunity 27: 647-659.

48. Simonian PL, Wehrmann F, Roark CL, et al. (2010): $\gamma \delta$ $\mathrm{T}$ cells protect against lung fibrosis via IL-22. J Exp Med 207: 2239-2253.

49. Nady S, Shata MT, Mohey MA, et al. (2017): Protective role of IL-22 against Schistosoma mansoni soluble egg antigen-induced granuloma in vitro. Parasite Immunol 39.

50. Park H, Li Z, Yang XO, et al. (2005): A distinct lineage of $\mathrm{CD} 4 \mathrm{~T}$ cells regulates tissue inflammation by producing interleukin 17. Nat Immunol 6: 1133-1141.

51. Broudy VC, Kaushansky K, Segal GM, et al. (1986): Tumor necrosis factor type alpha stimulates human endothelial cells to produce granulocyte/macrophage colony-stimulating factor. Proc Natl Acad Sci U S A 83: 7467-7471.

52. Jovanovic DV, Di Battista JA, Martel-Pelletier J, et al. (1998): IL-17 stimulates the production and expression of proinflammatory cytokines, IL-beta and TNF-alpha, by human macrophages. J Immunol 160: 3513-3521.

53. Moseley TA, Haudenschild DR, Rose L, et al. (2003): Interleukin-17 family and IL-17 receptors. Cytokine Growth Factor Rev 14: 155-174.

54. Lenardo MJ, Baltimore D (1989): NF-kappa B: a pleiotropic mediator of inducible and tissue-specific gene control. Cell 58: 227-229.

55. Siebenlist U, Franzoso G, Brown K (1994): Structure, regulation and function of NF-kappa B. Annu Rev Cell Biol 10: 405-455.

56. Dewas C, Dang PM, Gougerot-Pocidalo MA, et al. (2003): TNF-alpha induces phosphorylation of p47(phox) in human neutrophils: partial phosphorylation of p47phox is a common event of priming of human neutrophils by TNF-alpha and granulocyte-macrophage colony-stimulating factor. J Immunol 171: 4392-4398.

57. Wolk K, Kunz S, Witte E, et al. (2004): IL-22 increases the innate immunity of tissues. Immunity 21: 241-254.

58. Govan JR, Deretic V (1996): Microbial pathogenesis in cystic fibrosis: mucoid Pseudomonas aeruginosa and Burkholderia cepacia. Microbiol Rev 60: 539-574.

59. Brand S, Beigel F, Olszak T, et al. (2006): IL-22 is increased in active Crohn's disease and promotes proinflammatory gene expression and intestinal epithelial cell migration. Am J Physiol Gastrointest Liver Physiol 290: G827-G838. 